\title{
HIV Lipodystrophy
}

National Cancer Institute

\section{Source}

National Cancer Institute. HIV Lipodystrophy. NCI Thesaurus. Code C48899.

A neoplastic process characterized by a diffuse poorly circumscribed overg rowth of adipose tissue in the breast and cervical areas, and an accumulation of visceral fat. It is associated with antiretroviral therapy. Clinical presentation includes hyperlipidemia, insulin resistance, and fat atrophy of the face and limbs. 